\title{
Revista de la
}

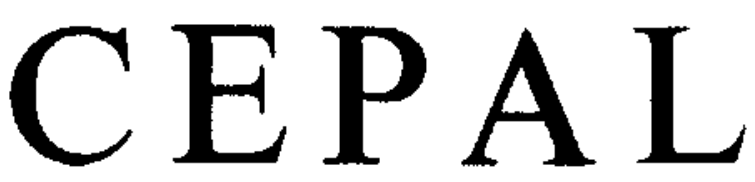

Director

\author{
RAUL PREBISCH
}

Secretario Técnico

A D O L F O GURR I ER I

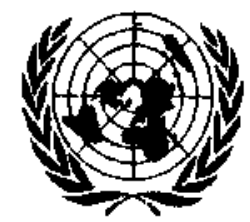

NACIONES UNIDAS

COMISION ECONOMICA PARA AMERICA LATINA

SANTIAGO DE CHILE / SEGUNDO SEMESTRE DE 1977 


\section{S U M A R I O}

La originalidad de la copia: la CEPA L y la idea de desarrollo

Femando H. Cardoso

Para 'otro desarrollo' : requisitos y proposiciones

Marshall Wolfe

Polftica fiscal y desarrollo integrado

69

Federico J. Herschel

Apuntes acerca del futuro de las democracias occidentales

José Medina Echavarría

Comentario de John Durston

Comentario de Carlo Geneletti

142

Comentario de Eduardo Palma

145

Comentario de Gregorio Weinberg

147

Comentario de Marshall Woife

150

Las pequefias naciones y el estilo de desarrolio 'constrictivo'

Carlos Real de Azúa

El déficit de los servicios urbanos: ¿una limitación estructural?

Francisco Barreto y Roy T. Gilbert

Sobre el artículo de Raúl Prebisch, "Crítica al capitalismo periférico"

Comentario de Joseph Hodara

Comentario de Eugenio Kossarey

Comentario đe Octavio Rodríguez

Comentario de Marshall Wolfe 
REVISTA DE LA CEPAL

Segundo Semestre de 1977

\section{El déficit de los} servicios urbanos: ¿una limitación estructural?

\section{Francisco Barreto $y$ Roy T. Gilbert*}

Los autores analizan las causas del déficit de servicios urbanos en Brasil y afirman que ellas encuentran su raiz en ta compleja combinación de una oferta inadecuada e insuficiente y una demanda perturbada por la crisis económica. Por un lado, subrayan que la oferta se articula a base de pautas tecnológicas y economicas inapropiadas a las necesidades y posibilidades de la población, pues no sólo se adoptan tecnologías propias de los países desarrollados, que en la realidad brasileña resultan demasiado complejas, rigidas y excluyentes, sino que la generación de la oferta se orienta por el criterio, propio de la empresa privada, que evalúa las inversiones por su rentabilidad. Por otso, seffalan que la situación económica crítica por la que atraviesa el pals ha afectado la capacidad de pago de la población de bajos ingresos, lo que impide que sus necesidades de servicios puedan convertirse en demanda efectiva. A esta profunda incoherencia entre oferta, demanda efectiva y necesidades básicas, se agregan los problemas financieros de los municipios y sus dificultades para realizar una acción programada.

Por consiguiente, sugieren que esa incoherencia sólo puede ser enfrentada mediante un cambio sustancial en los principios que orientan la oferta de servicios junto a una política redistributiva que aumente la capacidad de pago de la mayoría de la población.

*Funcionarios de la Subsede de la CEPAL en Brasil.

\section{Introdučción}

El Brasil atraviesa actualmente una etapa de urbanización explosiva; y el rápido crecimiento de la población urbana entraña un brusco aumento de las necesidades de infraestructura para atenderla.

De conformidad con lo señalado en el II Plan Nacional de Desarrollo, durante este decenio la población aumentará en aproximadamente 25 millones de habitantes, y habrá que crear las condiciones necesarias para que la oferta de servicios urbanos crezca a un ritmo similat. De este modo, servicios como abastecimiento de agua, recolección y trata miento de excretas humanas, energía, transporte, salud pública y asistencia médica hospitalaria, enseñanza, recreación, deportes, etc., constituirán el conjunto de la infraestructura básica necesaria.

Esta perspectiva se agrava cuando se considera que actualmente alrededor de $72.6 \%$ de las viviendas brasileñas no poseen agua potable, y sólo $12.1 \%$ están conectadas a un sistema general de alcantarillado. El déficit de estos servicios ilustra en forma amplia el desequili brio existente, el que incide fundamentalmente sobre la calidad de vida del habitante urbano del Brasil, sobre todo en los estratos de menores ingresos.

Además de este déficit ya existente, hubo un estancamiento relativo de la oferta, e incluso se produjo una verdadera involución de esta infraestructura en los principales centros del país, de acuerdo con el elevado índice de urbanización.

Conforme a estos planteamientos, este artículo procura caracterizar los principales aspectos relativos a la naturaleza de la crisis estructural, correspondiente al carácter del déficit de servicios urbanos enunciado. Así, se intentará examinar 
esta caracterización en dos momentos específicos: primero en el de la generación de la oferta, a través de la identificación del proceso $y$ de sus patrones determinantes; $y$, luego, en el de forma ción y exteriorización de la demanda de servicios en el medio urbano, cuya modalidad actual se analizará.

En estas dos partes principales se expondrán consideraciones teóricas se- guidas de un examen de las realidades concretas y se intentara comprender la verdadera naturaleza de las crisis identificadas.

Luego de estas consideraciones preliminares sobre los fundamentos de este trabajo, en las secciones siguientes se expondrán las principales hipótesis explicativas acerca de las causas del desequilibrio existente entre la oferta y la demanda de infraestructura urbana.

\section{A. \\ La oferta}

1. La generación de la oferta: el enfoque empresarial

Uno de los criterios más difundidos en la actualidad en el sector público, con relación a la oferta de equipos y servicios urbanos, es el principio de rentabilidad de la inversión pública, con el objeto de garantizar el rendimiento del capital invertido. Su justificación más frecuente consiste en sostener que la generación de servicios urbanos se incluiría en el proceso productivo general, por lo que debería implicar necesariamente una realimentación constante en términos del capital invertido.

Se adoptó este criterio luego de laber observado el comportamiento seguido por los países industrializados y sus experiencias en materia de instalat ción de equipos urbanos. En efecto, el principio de la rentabilidad financiera de la inversión prevaleció en la mayor parte de los países industrializados como una consecuencia natural del intenso proceso de industrialización que atravesaban.

Por ende, el proceso de instalación de equipos urbanos, según pautas empre- sariales, se sometió a las leyes internas del mercado, y en consecuencia a una intensa competencia económica y tecnológica. Así, la instalación de estos equipos, realizada aplicando criterios empresariales respondió a ciertos prerrequisitos económicos y financieros.

El desarrollo de los servicios urbanos no sólo acompatió el proceso de industrialización, sino que también recibió de éste el impulso necesario en el plano financiero y tecnológico.

Dentro de esta perspectiva, el principio de la maximización del lucro, apropiado para la inversión privada, se aplicó posteriormente en los de naturaleza pública, procurando evitar así la atomización de los recursos financieros, considerados escasos en proyectos no viables económicamente.

Más recientemente, el análisis de la relación costo-beneficio amplió el concepto de lucro, que para las inversiones públicas pasó a denominarse 'rentabilidad social'. De todas maneras, incluso admitido este aspecto ltamado 'social', se supone siempre la existencia de factores que podrian aumentar el producto nacio- 
nal reduciendo los 'costos sociales'. Así pues, este tipo de análisis sólo amplió las bases del criterio empresarial aunque sin alterar su verdadera naturaleza.

Por otra parte, si en los países industrializados, con la ampliación de este criterio se alcanzó un buen nivel de eficiencia, es difícil imaginar cómo podrían obtenerse resultados semejantes en el Brasil teniendo presente las condiciones actuales de la estructura económica interna.

Las tazones de esta discrepancia se apoyan, fundamentalmente, en las diferencias estructurales e históricas del actual proceso de industrialización y de urbanización entre dichos países.

La primera de estas diferencias se refiere al elevado costo de oportunidad, en la etapa actual, de la instalación de servicios urbanos en el Brasil. Los países industrializados pueden disponer de infraestructuras heredadas de situaciones anteriores, pero eso no ocurre en el grupo de paises llamados "en vias de desarro110". Para estos últimos, superar la carencia de servicios urbanos supondría exigir de las generaciones actuales un gran sacrificio, cuyos beneficios favorecerían esencialmente a las generaciones futuras, en tanto que en los paises industriali zados la generación actual es la que palpa estas ventajas.

Otra caracteristica se refiere a los patrones socioeconómicos (estructura del ingreso, del consumo, estratos sociales, valores culturales, etc.) que aparecen como sumamente diferenciados en los paises carentes de infraestructura urbana, mientras que muestran una relativa homogeneidad en los países industrializados.

De modo más concreto, se puede observar que los paises no industrializados carecen de tecnologias propias, $y$ cuando las poseen no las utilizan. A modo de ejemplo, podría señalarse el caso de la taipa' un material tradicional de construcción usado especialmente en el nordeste del Brasil, y que sin embargo no ha sido admitido oficialmente por las entidades que desarrollan actividades relacionadas con la vivienda popular. Como contrapartida, baste señalar que se importan tecnologías que, desde el punto de vista económico, ecológico, o de ambos, suelen no adaptarse a las condiciones locales.

Por ultimo, cabe destacar un hecho básico que caractëriza a los diferentes grupos de estos palses: la estructura del ingreso. En efecto, los bajos niveles de ingreso de la mayoría de la población en los países no industrializados, genera una incapacidad generalizada de pagar los servicios necesarios. Esto a su vez, se convierte en una justificación para que, con arreglo al criterio empresarial, no haya oferta.

De donde una proporción importante de la responsabilidad de dicha oferta se concentra en el sector público, al comprobarse en estos países una escasa participación del sector privado en la instalación de equipos urbanos.

Por tanto, cabe suponer que la incorporación de actitudes y valores basados en patrones socioeconómicós y técnicos ajenos a la realidad brasileña, podrá intensificar aún más las causas de una crisis de la oferta de servícios urbanos que, desde ya, asume proporciones casi irreversibles. Así, el grado de compatibilidad entre el criterio empresarial con el desarrollo de la oferta de servicios urbanos, mucho depende de la estructura económica y social del país.

\footnotetext{
'Taipa: pared hecha de arcilla, la que puede contener incluso cal y arena, mezcladas con trozos de madera. Se destaca que esta tecnología fue aplicada en las construcciones de la Época colonial brasileña (siglo XVIII) y resisten hasta el presente.
} 
2. Imitación de patrones socioeconómicos y técnicos

La imitación de patrones socioeconómicos y técnicos es una constante en la generación de la oferta de equipos urbanos. Se puede observar, por el tipo de construcción $\mathrm{y}$ por las modalidades de los servicios urbanos considerados necesarios (como ocurte con la prioridad atribuida a los automóviles mediante la instalación de la red vial urbana), que los patrones adoptados generalmente son importados, adaptados a una demanda más homogénea similar a la existente en los países industrializados.

Asi, se comprueba que la generación de la oferta de servicios urbanos basada en el criterio empresarial, deriva de patrones extraños a la realidad local, lo que conduce casi siempre a situaciones criticas. Esto puede atribuirse a la falta de capacidad de pago de la población, desde el punto de vista de la demanda, y también, a las limitaciones financieras locales desde el punto de vista de la oferta.

De este modo se comprueba que el proceso actual de desartollo y modernización de los equipos urbanos, pone de relieve los intentos de alcanzar, cualita tiva y cuantitativamente, los mismos pa trones de los países industrializados; así, entre otros, se incluye por ejemplo la reconocida adecuación de las ciudades al au tomóvil a través de grandes inversiones en infraestructura vial, como ocurre con los viaductos, túneles, etc.

$Y$ por otro lado, se desconoce la importancia de las tecnologfas autóctonas, como en el caso de las viviendas espontáneas entre las que destacan las 'favelas' de las grandes ciudades. En estas comunidades se observa la creación de soluciones técnicas colectivas, como por ejemplo, construcciones con materiales improvisados, la ubicación estratégica de las fuentes de agua, e incluso sistemas au tónomos de recolección de residuos; $y$ por último, la elaborada organización de la comunidad basada en la autogestión que suponen dichas soluciones. Aunque creadas en condiciones difficiles, estas tecnologias deberian ser evaluadas con miras a su eventual incorporación por la oferta oficial de servicios urbanos, de la cual aunque, informalmente, ya constituyen una parte importante.

Si se examinan los patrones tecnoló gicos adoptados en la actual oferta de servicios urbanos, se observa que éstos constituyen por sí mismos un factor discriminatorio, y que a priori, limitan mediante parámetros bien precisos el universo de la oferta que será generada. En otras palabras, resta saber cómo podrá la oferta satisfacer las necesidades de la po blación de bajos ingresos, si desde un comienzo los patrones que la delimitarán son, por su naturaleza tecnológica, rígidos y excluyentes.

Por otra parte, la adopción de tecnolo. gías complejas implica exigir una programación igualmente compleja; y por programación se entiende aquí la capacidad que posee el gobierno local de planificar sus actividades de inversión, con miras a la realización de un programa de trabajo definido. Y como la programación está vinculada a un trabajo intenso de encuestas para conocer las necesidades locales, que se anticipan a una respuesta concreta a los anhelos de la población, constituye una etapa fundamental en la generación de la oferta de servicios urbanos. Por consiguiente es cuestionable aquella modalidad de programación que utiliza informaciones originarias de los estratos superiores, sin un respaldo técnico efectivo o una verificación empírica.

Además, cabe señalar que casi no hay recursos humanos locales técnicamente capaces de programar la oferta de servi- 
cios urbanos. Se observa que, por regla general, los servicios que poseen una mejor infraestructura programática son precisamente los que dependen de la asignación de recursos externos y que exigen la incorporación de una capacidad operativa.

En la actualidad, se observa que la mayor parte de las municipalidades brasi lenas carecen de las informaciones mínimas necesarias para una programación correcta. En especial, se cita la falta de archivos de trabajo con informaciones sobre los proyectos realizados y el orden de ejecución de los proyectos por realizar. En efecto, disponer de dichos instrumentos significaría un avance importante, no sólo para comprender cómo desarrollar mejor las actividades, sino también cómo prever mejores alternativas.

Si en determinados casos la carencia de la programación constituye un obstáculo para el desarrollo armónico de la oferta y la demanda, en otros (admitida una cierta capacidad instalada) puede incidir sobre las ya limitadas finanzas municipales, hasta llevarlas a su agotamiento. En estas condiciones, cabe suponer que una buena capacidad de programa ción también podrá estrangular las finanzas locales, si hace un uso intenso de la capacidad de endeudamiento existente.

Este aspecto demostraría en forma clara que desde el punto de vista de la programación, una solución parcial no producirá resultados satisfactorios. Se estima que es necesario atender simultáneamente a los problemas de carácter financiero, para mejorar la capacidad de recaudación, así como a los derivados de la captación de la demanda potencial de servicios urbanos.

\section{3. ¿Crisis financiera o crisis económica?}

Conforme al criterio de análisis seguido hasta aquí, se observa que la crisis de la oferta de servicios urbanos, tiende a agravarse cuando se aplica un punto de vista empresarial, que adopta patrones socioeconómicos y técnicos derivados de otras realidades. Actualmente en el Brasil esta crisis se manifiesta en el agotamiento de los recursos financieros locales disponibles para invertir en la infraestructura urbana de la mayoria de las municipalidades brasileñas. Con frecuencia, éstas dependen de transferencias y préstamos recibidos de otras instancias; y en el caso de estos últimos, dada su baja capacidad de endeudamiento, se les hace imposible recurrir a ellos.

En realidad, la presente crisis financiera está profundamente vinculada a una crisis más amplia que afecta a la estructura económica del país. Por ende, cabe suponer que las ineficiencias señala das de los sistemas locales de recauda ción son sólo expedientes parciales e incompletos, para explicar las actuales limitaciones financieras del conjunto de los municipios brasileños.

En virtud de lo antecedente, se comprende que no siempre una capacidad suficiente de recursos es función exclusiva de una buena máquina recaudadora. Además, es sabido que la recaudación de cualquier municipalidad puede encontrar barreras que restringen la expansión de las finanzas locales; una de ellas, la reducida capacidad económica de la población es una limitación de naturaleza estructural. Esta incapacidad relativa de la población se encuentra condicionada, tanto por la estructura del ingreso, como por su grado de participación en el proceso productivo general, el que puede estimarse por la tasa de empleo. 
De la situación expuesta cabe deducir dos conclusiones básicas: la primera, que ante la falta de recursos en la esfera local, debe hacerse necesariamente referencia a una situación más amplia, es decir, la socioeconómica; y en segundo lugar, se estima que las respuestas que serán evaluadas, condicionadas por los patrones adoptados según el criterio empresarial, agudizan una situación fi- nanciera precaria en función de los altos costos de dichos patrones.

Por último, bajo la apariencia financiera, se encubren en gran parte situaciones crónicas desde el punto de vista económico, de modo que el sistema muestra una incapacidad financiera y no puede ofrecer con rapidez respuestas que satisfagan las necesidades de servicios urbanos de los estratos de bajos ingresos.

B.

Demanda

1. Formación de la demanda; aspectos teóricos y prácticos

Hasta aquí se analizaron, desde el punto de vista de las necesidades de la población, los elementos relevantes de la oferta. En las secciones siguientes se examinarán esos elementos en función de los aspectos que hacen a la formación de la dentanda de servicios urbanos.

Antes de proseguir, y para mejor caracterizar la demianda de servicios urbanos, conviene definirla. Por demanda se entiende el conjunto de necesidades que una determinada población considera imprescindible para la realización de una vida urbana compatible con sus aspiraciones.

Este concepto de necesidades pone de relieve las aspiraciones de la comunidad $y$, en consecuencia, subraya la importancia del análisis del proceso de formación de la demanda. Cabe destacar que dentro del conjunto de la demanda se servicios urbanos, resulta fundamental establecer dos distinciones importantes relativas a su formación. La primera de ellas se relaciona con la estructura del ingreso, y la otra se refiere a la propia oferta según sus patrones limitativos.

De esta manera, se reconoce la existencia de una demanda potencial, que representa el conjunto de las necesidades $y$ aspiraciones de la población en materia de equipos y servicios urbanos, y que no se encuentra necesariamente cnunciada o cuantificada con claridad.

Dentro de esta perspectiva, la demanda potencial se transfonna en demanda efectiva de acuerdo a la capacidad de pago de la población, y por lo tanto, las poblaciones de bajos ingresos tienen escasas posibilidades de ver satisfecha siquiera una parte mínima de la demanda potencial.

En realidad, para la población de los estratos de ingresos más elevados la diferencia entre demanda potencial y efectiva rara vez se plantea, ya que para ellos la demanda potencial es casi siempre efectiva. Para las demás categorías esto casi nunca ocurre, y se trata de una distinción fundamental derivada de la estructura del ingreso.

Debe tenerse presente que según el criterio empresarial si no existe una de- 
manda efectiva no se justificaría una oferta correspondiente. Por estos motivos competería entonces a los organismos que prestan servicios urbanos, captar la demanda potencial de la población de bajos ingresos. A través de sus diversos instrumentos técnicos y políticos, el sector público trata de 'captar' la demanda, basándose en el criterio empresarial y en sus patrones tecnológicos.

De esta forma, se puede considerar que la demanda 'captada' es la versión oficial de la demanda potencial y, al mismo tiempo, de la demanda efectiva. Esta versión oficial excluye, a priori, los elementos derivados de los patrones socioeconómicos y técnicos de la población, no reconocidos oficialmente por los organismos prestadores de servicios.

En consecuencia, se comprueba así la existencia de una segunda distinción fundamental en la formación de la demanda, la que se refiere a la propia estructura limitativa de la oferta actual de servicios urbanos. Esta limitación se impone básicamente sobre las poblaciones de bajos ingresos, si bien hay escasa diferencia entre la demanda potencial y la demanda captada de la población de rentas medias y altas. Esto obedece al liecho de que los patrones de estos estratos son precisamente los adoptados of icialmente y apli cados en forma general para satisfacer la demanda de servicios urbanos.

Para el resto de las categorías sociales, las divergencias son tan importantes que se justificaría afirmar que en estos diversos aspectos de la demanda existen indicios de una porfunda crisis.

\section{Patrones socioeconómicos e institu- cionales}

Es un hecho muy conocido en los medios técnicos, que la captación de la demanda potencial, es decir, el proceso en virtud del cual se definen las demandas prioritarias para los agentes del poder, es función directa del proceso decisorio local. Así, la dirección hacia la cual se orientará la oferta, quedará establecida como resultado de un proceso de múltiples presiones o reivindicaciones en el que intervienen instituciones que, formal o informalmente, integran la vida política local. En consecuencia, la insta lación o ampliación de servicios urbanos, no es sino una respuesta natural y recíproca an te este sistema de presiones.

Fácil es advertir que la demanda de esta manera percibida, derivada precisamente de un proceso de presiones, y como es evidente de la capacidad que poseen los diversos sectores de la población de exponer con claridad sus necesidades en materia de infraestructura urbana y exigir que ellas sean satisfechas.

Por tanto, el actual mecanismo de captación de la demanda potencial de servicios urbanos expresa no solamente al sistema de influencias que componen el proceso decisorio, sino que también es la resultante de la existencia de canales oficiales de comunicación entre quienes ejercen el poder y la comunidad.

Este sistema de presiones que se ejerce a través de los diversos cauces de comunicación existentes, brinda información al gobierno local, a los organismos prestatarios de servicios urbanos o a ambos, $y$ sirve como elemento de juicio para la adopción de decisiones. De esta manera, la demanda potencial sólo puede ser captada debidamente a través de dichas instituciones, las que trasmiten las reclamaciones y permiten así la articulación entre quienes demandan y quienes ofrecen servicios urbanos. Se debe destacar que una de las modalidades más frecuentes y tradicionales de captación de la demanda potencial está vinculada a los canales políticos, como ocurre en el caso 
específico del ejercicio del cargo de edil, quien es principalmente un portavoz de las necesidades más inmediatas de la población. Si bien casi siempre la informa ción transmitida de esta forma posee una elevada connotación política (y tampoco podfa dejar de tenerla), es una de las vias más activas de que dispone la población para hacerse escuchar en los niveles de decisión.

Este tipo de representatividad está más al alcance de los individuos situados en los tramos superiores de ingresos (equivalentes al nivel de los países industrializados). Como consecuencia de ello se observa que los estratos más pobres de la población (precisamente los más necesitados) son justamente los que tropiezan con mayores dificultades para poner de manifiesto la demanda y además, no tienen acceso a este tipo de representatividad. Esta evidente marginalización obedece a su posición económica y a su escasa importancia política (medida en función de votos efectivos, ya que el analfabetismo todavía constituye un factor considerable).

Esta situación genera una verdadera distorsión de la captación de las aspira ciones y necesidades de importantes sectores de la población urbana brasilena; distorsión que contribuye en forma clara a aumentar la distancia entre la oferta y la demanda de la población de bajos ingresos.

La falta de instrumentos formales a disposición de estos estratos de la población, los llevó a organizarse espontáneamente en asociaciones comunitarias de barrios. Ahora bien, considerando que el equipamiento urbano y los servicios conexos constituyen un bien colectivo, estas asociaciones surgen como una modalidad importante de organización de la colectividad en el proceso de formación y expresión de su demanda. Los agentes del poder pueden de este modo, tener a su alcance un instrumento eficiente de información, el que podrá proporcionar un cuadro fiel del consenso de la pobla ción sobre sus necesidades y aspiraciones más apremiantes. La utilidad de las asociaciones comunitarias es comprensible ya que pueden asegurar, tanto al gobierno local como a los organismos prestatarios de servicios, informaciones basadas en las necesidades efectivas de una población, cuya demanda potencial tiene pocas probabilidades de transformarse en demanda efectiva.

La experiencia de algunas ciudades brasileñas en materia de desarrollo comtnitario, corrobora lo anterior. En consecuencia, el desarrollo de este tipo de asociaciones de barrio en los centros urbanos donde aún perduran, demuestra que su eventual afianzamiento, y el hábito de consultarlas adquirido por gobiernos locales, lejos de ser negativo para ellos, no sólo es muy positivo, sino muy conveniente desde el punto de vista de la demanda.

Además, los alcaldes y sus representantes pueden enterarse, a través de estas asociaciones, de las necesidades de la población. Por último el diálogo entre el gobierno local y la comunidad es una valiosa innovación que si bien comporta riesgos posee ventajas indiscutibles.

3. ¿Crisis económica o crisis de los patrones?

Además de la ya señalada tendencia a una crisis de la oferta, desde el punto de vista de la demanda existen dos problemas fundamentales que llevan a las poblaciones de bajos ingresos a una situación crítica.

El primero se refiere a su propia estructura económica que imposibilita la transformación de la demanda potencial 
en demanda efectiva, dada la incapacidad en que están esas poblaciones para pagar los servicios urbanos prestados. El segundo aspecto se refiere a los patrones oficiales de la oferta, es decir, al desajuste entre la demanda potencial (o efectiva) y la demanda captada.

En el primer caso, se trata de una situación íntimamente vinculada a la condición económica individual. Esto significa que a partir de una oferta generada según criterios empresariales, la demanda efectiva asume una importancia capital en la atracción de la oferta corres pondiente. Sólo quienes tengan ingresos medios y altos están en condiciones de pagar los servicios urbanos, como puede demostrarlo el cuadro siguiente.

Según el cuadro anterior, se comprueba que la proporción de viviendas, en 1970. con agua y alcantarillado en cada tramo de ingreso aumenta progresivamente en razón directa al nivel de ingre. so. Además, se puede observar que, sólo en los tramos de ingresos más altos (a partir de 3000 cruceiros mensuales) la proporción de las viviendas abastecidas de agua, respectivamente $76.3 \%$, $84.10 \%$ y $89.3 \%$, alcanza los niveles actuales de servicios de los países más desarrollados. No es casualidad que en estos tramos de ingreso (por sobre 3000 cruceiros) se encuentre precisamente la casi totalidad de las viviendas de los paises industrializados, ${ }^{2}$ en tanto que en el Brasil el porcentaje es mínimo en estos tramos.

\footnotetext{
${ }^{2}$ Aunque subsista una relativa dificultad en este tipo de comparaciones, sín embargo puede afirmarse que aproximadamente para el mismo año el 90\% de las viviendas de Gran Bretaña estaban en los tramos de ingreso de $3000 \mathrm{cru}$ ceiros o más. (Véase Central Statistical Office, Annual A bstract of Statistics,H.M.S.O., Londres, 1974.)
}

Puede observarse en la actualidad que las viviendas con un buen servicio de agua son precisamente aquellas que corres ponden a los tramos superiores de ingresos. Por otra parte, también para los servicios de alcantarillado se advierte idéntico desequilibrio entre los diversos estratos de la población. Asimismo, se observa que la proporción de viviendas atendidas por este servicio es bastante inferior a las que disponen de servicio de agua. Esto se explica por la inclusión de viviendas rurales, que no necesitan ese servicio, cualquiera que sea su nivel de ingresos.

Si se supone que la situación es semejante para los demás servicios que integran la infraestructura urbana, podría afirmarse la existencia de una grave crisis que económicamente se pone de mani fiesto en la incapacidad de transformar la demanda potencial existente en demanda efectiva.

La denominada crisis económica se vería así agravada por los actuales patrones de oferta y por el proceso de definición de la demanda captada. En consecuencia, como ya se expresó, los elevados costos derivados de tecnologías complejas y de las dificultades de su incorporación al plano local, colocan a esos patrones muy por encima de las posibilidades económicas de la mayor parte de la población.

La naturaleza discriminatoria de los patrones tecnológicos utilizados impide, en cierto sentido, que se apliquen soluciones oficialmente no admitidas desalentándolas así desde el punto de vista de su eficiencia técnica, lo que incide sobre la propia eficiencia de las tecnologias locales, dificultando su proceso evolutivo. Así, puede observarse la escasa evolución de la técnica de pozos sépticos y simples, como medio de solucionar el problema de eliminación de los detritos 


\section{BRASIL: DEMANDA EFECTIVA DE SERVICIOS DE ABASTECIMIENTO DE AGUA Y ALCANTARILLADO SEGUN LOS DIVERSOS TRAMOS DE INGRESO, 1970}

\begin{tabular}{|c|c|c|c|}
\hline \multirow[b]{2}{*}{$\begin{array}{c}\text { mareso mensual } \\
\text { del hogar } \\
\text { (kin (ruceiros) }\end{array}$} & \multirow[b]{2}{*}{$\begin{array}{l}\text { Número de } \\
\text { hogares } \\
\text { (Én miles) }\end{array}$} & \multicolumn{2}{|c|}{ En cada traino de ingreso } \\
\hline & & $\begin{array}{c}\text { Porcentaje del } \\
\text { total con abas- } \\
\text { tecimiento de } \\
\text { asua b }\end{array}$ & $\begin{array}{l}\text { Porcentaje det } \\
\text { total con alcan- } \\
\text { tarillado }\end{array}$ \\
\hline Hasta - $\quad 150$ & 979 & 2.1 & 0.7 \\
\hline $151 \cdot 300$ & 2747 & 3.1 & 1.1 \\
\hline $301-450$ & 2162 & 7.0 & 2.6 \\
\hline $451 \cdot 600$ & 2281 & 14.6 & 6.2 \\
\hline $601-750$ & 983 & 18.6 & 7.8 \\
\hline $751-900$ & 1174 & 25.6 & 11.1 \\
\hline $901-1200$ & 1394 & 33.1 & 14.8 \\
\hline $1201 \cdot 1500$ & 1039 & 42.9 & 19.9 \\
\hline $1501-3000$ & 2198 & 58.2 & 28.5 \\
\hline $3001 \cdot 4500$ & 692 & 76.3 & 40.5 \\
\hline $4501-6000$ & 340 & 84.1 & 46.8 \\
\hline+ de -6000 & 543 & 89.3 & 51.2 \\
\hline Sin declaración de ingresos & 1097 & $\cdot$ & $\cdot$ \\
\hline Total & 17629 & 27.4 & 12.1 \\
\hline
\end{tabular}

Fuente: IBGE, VIII Recenseamento geral, 1970. Guanabara, junio de 1971.

A precios de 1976 .

bPor abastecimiento de agua se entiende una red general y conexión interna a Jos domicilios. cPor alcantarillado se entiende que las viviendas $\$$ encuentran conectadas a una red general.

humanos. Si bien este método es sanitariamente seguro cuando la densidad demográlica es baja y se respetan debidamente los cursos de agua, oficialmente no se estimula su aplicación pues no satisfacc la imagen progresista que el sector dominante de la sociedad desea promover a través de la oferta de servicios urbanos.

En vez de considerar soluciones de csta índole actualmente utilizadas por la población de escasos recursos, como posibilidades concretas para eliminar el déficit de algunos servicios urbanos, se las considera más bien como problemas.

De esta manera, la imposibilidad de captar la demanda potenciall (y la de las diversas modalidades existentes que reflejen un alto grado de creatividad) dificulta aún más la compleja tarca de mejorar la calidad de vida de la población urbana brasileña. 


\section{Conclusiones}

El análisis de la oferta y demanda de servicios urbanos constituye, sin duda, un asunto extremadamente complejo, por otra parte imposible de abarcar en un trabajo tan breve como el presente. Son innumerables las dudas que persisten, y las conclusiones aquí enunciadas se refieren a un conjunto de hipótesis y, como tales, sujetas a revisión crítica. En forma preliminar, y habida cuenta de estos límites, se puede, en una primera aproximación a estos problemas, sugerir algunas conclusiones.

Desde el punto de vista de la oferta de servicios urbanos existe una situación crítica. $Y$ con frecuencia se invocan razones de carácter financiero para justificar el verdadero carácter de esta crisis. Ahora bien, desde el punto de vista coyuntural este tipo de explicaciones es satisfactorio, pero se trata de una visión parcial de una crisis más profunda, la económica.

Se advirtió asimismo que la crisis económica constituye la causa principal de la situación crítica por la que atraviesa la demanda. Por eso cuando se examina la verdadera naturaleza del desequilibrio entre la oferta y la demanda de servicios urbor nos en la mayoría de las ciudades brasilenas sería imposible dejar de tomar en cuenta una crisis estructural.

En consecuenciá, uno de los propósitos principales de este trabajo, fue demostrar que existe esta situación critica que afecta a la demanda, por su limitada capacidad de pago, y que ésta se opone a la adopción de un criterio empresarial para abordar la oferta de servicios urba- nos. Se perfila de esta manera un verdadero callejón sin salida, deterninado ya sea por la incapacidad económica de la población o por la necesidad que tienen los servicios públicos de reponer sus existencias de capital.

En efecto, la actual estructura econó mica brasileña (considerada desde el punto de vista de la situación actual de las poblaciones de bajos ingresos), no es compatible con una oferta de servicios urbanos generada según criterios empresariales y que, además, adopta patrones tecnológicos apropiados para países industrializados.

La incompatibilidad primordial apa rece en los organismos locales prestadores de servicios, incapacitados de desarrollar programas de inversiones, sea con recursos propios o no. Esto significa que dichos organismos ni siquiera pueden recurrir a fuentes externas de financiamiento (dada su baja capacidad de endeudamiento) y además tampoco están en condiciones de actuar aplicando esos patrones tecnológicos.

De csta manera, se admite que incluso aunque se superara esta crisis de recursos, con un rápido aumento de las disponibilidades financieras locales, las municipalidades tropezarían con otro obstáculo: la carencia de capacidad para programar. ejecutar y llevar a cabo proyectos aplicando patrones copiados de otras realidades.

Por ello, debe destacarse que el desconocimiento de otras tecnologías adaptadas a las condiciones locales, refuerza ampliamente la crisis financiera, dados 
los elevados costos de los patrones tecnológicos empleados por las instituciones oficiales.

En las esferas técnicas suele propo nerse para solucionar esta crisis, elevar rápidamente la capacidad de pago de las poblaciones de bajos ingresos, a través de un aumento del producto bruto global. Incluso aunque se produjese el mencionado aumento, parece poco probable que las poblaciones lleguen a poseer una capacidad de pago suficiente como para expresarse en demanda efectiva, dados los parámetros actuales del modelo. En este sentido, el sector público tiene la responsabilidad fundamental de corregir las deformaciones que se encuentran en la generación de la oferta de estos servicios.

Por otra parte, los estratos de la población que disfrutan actualmente en el Brasil de servicios urbanos satisfactorios son precisamente aquellos que poseen un nivel de ingresos más elevado; y se hallan en una situación semejante a la de los estratos sociales equivalentes de los paises industrializados, de donde provienen los patrones oficiales de oferta de dichos servicios.

En consecuencia, se estima fundamental que se elaboren programas globales para la oferta actual de servicios urbanos, que consideren innovaciones tecnológicas compatibles con las diferencias internas entre la realidad social y económica de la población urbana considerada como un todo. En este sentido es importante basar dichas innovaciones sobre la valiosa experiencia de las comunidades, como ocurre con la construcción de casas propias sin el apoyo de instituciones oficiales, y hasta con organización espontánea de los propios servicios de agua y alcantarillado y limpieza urbana. Dicha revisión de los patrones oficiales significaría el reconocimiento de la heterogeneidad estructural de la sociedad brasileña que se expresa en términos económicos, sociales, culturales, etc.

Dentro de esta perspectiva es preponderante el papel de las asociaciones comunitarias como elemento de referencia para la ampliación de los patrones tecnológicos y de sus soluciones alternativas. Ellas no sólo pueden transmitir las necesidades reales de las poblaciones de bajos ingresos sino también sus peculiaridades culturales. Además, dichas asociaciones pueden desempeñar un papel importante en la definición de los diversos tipos de tecnologias adecuados para cada caso, en consonancia con las experiencias existentes.

Así, se estima que sólo a través de la captación directa de las aspiraciones reales de las poblaciones de bajos ingresos por parte de las instituciones prestadoras de servicios se podrán dar los pri-. meros pasos para superar la crisis actual.

Tórnase evidente entonces la necesidad de realizar cuanto antes un amplio estudio de la demanda potencial y efectiva de dichas poblaciones, teniendo en cuenta una revisión probable de los supuestos del criterio empresarial $y$, en consecuencia, de los patrones adoptados. De esta manera, con el establecimiento de metas sobre la base de la realidad concreta, se podrán formular las respuestas apropiadas al gran desafio planteado, que es el mejoramiento de la calidad de vida de los habitantes de bajos ingresos de las ciudades brasileñas. 